\title{
Caught at its own game: regulatory small RNA inactivated by an inducible transcript mimicking its target
}

\author{
Nara Figueroa-Bossi, Martina Valentini, ${ }^{1}$ Laurette Malleret, and Lionello Bossi ${ }^{2}$ \\ Centre de Génétique Moléculaire, Centre National de la Recherche Scientifique (CNRS), Gif-sur-Yvette 91198, France
}

\begin{abstract}
A relevant, yet little recognized feature of antisense regulation is the possibility of switching roles between regulatory and regulated RNAs. Here we show that induction of a Salmonella gene relies on the conversion of a small RNA from effector to regulatory target. The chiP gene (formerly $y b f M$ ), identified and characterized in the present study, encodes a conserved enterobacterial chitoporin required for uptake of chitin-derived oligosaccharides. In the absence of inducer, chiP is kept silent by the action of a constitutively made small RNA, ChiX (formerly SroB, RybC), which pairs with a sequence at the $5^{\prime}$ end of chiP mRNA. Silencing is relieved in the presence of chitooligosaccharides due to the accumulation of an RNA that pairs with ChiX and promotes its degradation. Anti-ChiX RNA originates from an intercistronic region of the chb operon, which comprises genes for chitooligosaccharide metabolism and whose transcription is activated in the presence of these sugars. We present evidence suggesting that the chb RNA destabilizes ChiX sRNA by invading the stem of its transcription terminator hairpin. Overall, our findings blur the distinction between effector and target in sRNA regulation, raising the possibility that some of the currently defined targets could actually be inhibitors of sRNA function.
\end{abstract}

[Keywords: Antisense regulation; chitoporin; chitobiose; $\mathrm{Hfq}_{\text {; }}$ small RNA]

Supplemental material is available at http://www.genesdev.org.

Received March 31, 2009; revised version accepted July 9, 2009.

In the last decade, the study of a growing number of noncoding small RNAs in Escherichia coli and other bacteria has unveiled unsuspected layers of regulation in a variety of cell processes and responses (Gottesman et al. 2006; Vogel 2009; Waters and Storz 2009). The majority of bacterial sRNAs characterized to date are translational regulators, some binding translational regulatory proteins (Babitzke and Romeo 2007; Lapouge et al. 2008); others directly targeting messenger RNAs (mRNAs) (Storz et al. 2004; Aiba 2007). Members of the latter class act by base-pairing with complementary sequences in the proximity of the translation initiation site. In most instances, formation of the RNA duplextypically $<20$ base pairs long-sterically interferes with ribosome loading onto the mRNA (Udekwu et al. 2005; Morita et al. 2006; Bouvier et al. 2008). This inhibits translation initiation, rendering the mRNA more susceptible to RNase attack and decay (Massé et al. 2003; Moll et al. 2003; Morita et al. 2005; Rasmussen et al. 2005; Udekwu et al. 2005). There are also cases in which pairing

\footnotetext{
${ }^{1}$ Present address: Département de Microbiologie Fondamentale, Université de Lausanne, CH-1015 Lausanne, Switzerland.

${ }^{2}$ Corresponding author.

E-MAIL bossi@cgm.cnrs-gif.fr; FAX 33-1-69823811.

Article published online ahead of print. Article and publication date are online at http://www.genesdev.org/cgi/doi/10.1101/gad.541609.
}

by the sRNA prevents the formation of alternative secondary structures that themselves inhibit ribosome binding. In these situations, the interaction with the sRNA stimulates translation (Majdalani et al. 1998; Majdalani et al. 2005).

The mRNA:sRNA association is assisted by chaperon protein $\mathrm{Hfq}$, which facilitates the encounter/association with the mRNA target and, in some cases, protects the sRNA from degradation (Zhang et al. 2002; Geissmann and Touati 2004). Hfq is thought to bind to AU-rich regions in both the sRNA and its target (Brennan and Link 2007). Mutants lacking Hfq have been isolated in a number of bacterial species; although viable, these strains are debilitated and more susceptible than wild type to stress treatments. Such impairments have multiple causes including the inability of $h f q$ mutants to fully induce the RpoS-dependent general stress response, due to the loss of sRNA-mediated translational activation of rpoS mRNA (Brown and Elliott 1996; Majdalani et al. 1998), and a toxic overexpression of the RpoE regulon ascribable to loss of sRNA-mediated envelope homeostasis (Ding et al. 2004; Figueroa-Bossi et al. 2006; Guisbert et al. 2007; Sittka et al. 2007).

Regulatory sRNAs are thought to play a major role in improving bacterial adaptation to environmental changes. A significant portion of sRNA regulatory activity in 
E. coli and Salmonella is devoted to modulating the protein composition of the outer membrane, the compartment most directly involved in exchanges with the environment (Guillier et al. 2006; Vogel and Papenfort 2006; Valentin-Hansen et al. 2007). All of the major porins and several outer membrane proteins (OMPs) are targets for regulation by one or more sRNAs. The genes encoding these sRNAs are generally regulated at the transcriptional level, and expressed under specific conditions (Mizuno et al. 1984; Chen et al. 2004; Douchin et al. 2006; Figueroa-Bossi et al. 2006; Papenfort et al. 2006; Bossi and Figueroa-Bossi 2007; Thompson et al. 2007; Udekwu and Wagner 2007; Rasmussen et al. 2009).

An interesting feature in the working mechanism of some sRNAs emerged from the study of RyhB, an sRNA that accumulates in response to iron limitation and down-regulates a number of genes for iron-binding proteins (Massé and Gottesman 2002). RyhB was found to be "consumed" when active in regulation. Apparently, upon pairing with the target mRNA, RyhB sRNA is no longer protected by Hfq and becomes susceptible to degradation by RNase E (Massé et al. 2003). Thus, the RyhB-mediated response relies on the continuous production of the sRNA, which permits rapid reversal of the response when iron concentration increases. The same study showed other sRNAs to act by a similar stoichiometric mechanism (Massé et al. 2003).

In the present study, we identify a novel OMP, ChiP, involved in the uptake of chitin-derived oligosaccharides. The protein, required for Salmonella to grow on chitooligosaccharides as sole sources of both carbon and nitrogen, is only synthesized in the presence of its substrates. We show that ChiP synthesis results from destabilization of a constitutively made small RNA, ChiX, that normally represses ChiP synthesis by pairing with a sequence in the $5^{\prime}$ untranslated region (UTR) of chiP mRNA. This regulatory response occurs by a novel mechanism involving the production of an RNA sequence that can act as an alternative pairing partner for ChiX sRNA. This alternative base pair interaction is ill fated for ChiX sRNA as it targets the sRNA for nucleolytic decay.

\section{Results}

\section{Novel sRNA-regulated OMP in Salmonella}

To identify potential targets for regulatory sRNAs in Salmonella, in a previous study we screened a library of random MudK(lac) chromosomal insertions, searching for isolates whose lacZ levels varied in the presence or absence of Hfq. One fusion, strongly up-regulated in the $\Delta h f q$ background, had the MudK element inserted in the uncharacterized ybfM locus (Fig. 1A; Figueroa-Bossi et al. 2006). Analysis of Salmonella OMP preparations in polyacrylamide gels showed that the extract from the $\Delta h f q$ strain included a band that was missing in the wild-type sample (Fig. 1B). When eluted from the gel and subjected to MALDI-TOF analysis, the protein was found to correspond to YbfM. Subsequent analysis (described below) revealed that YbfM is a chitoporin; hence, it was renamed ChiP.

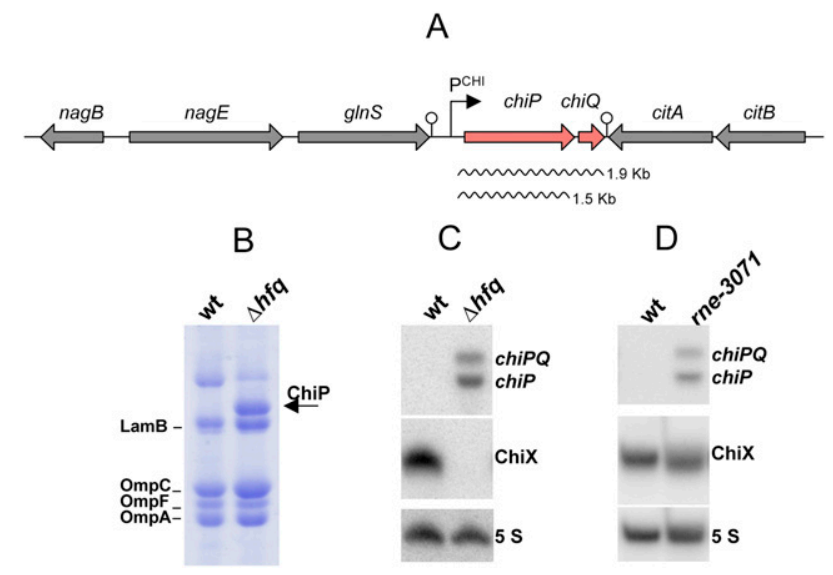

Figure 1. Small RNA-mediated silencing of a Salmonella omp gene. (A) Schematic diagram showing the gene organization of the segment between bp 744131 and 753872 of the Salmonella chromosome (McClelland et al. 2001). The transcription pattern of $c h i P(y b f M)$ and chiQ (ybfN) genes was established by Northern blot hybridization with promoter-proximal and promoterdistal probes (pp977 and pA83, respectively) (see the Supplemental Material). (B) Analysis of OMP preparations from wildtype and $\Delta h f q$ mutant strains. Cultures of strains MA8740 (ompD::Tn10 $h f q^{+}$) and MA8741 (ompD::Tn10 $\Delta h f q$ ) were grown in $\mathrm{LB}$ to an $\mathrm{OD}_{600}$ of 0.35 and processed for the OMP preparation. $(C, D)$ Northern blot analysis of chiPQ and ChiX RNAs from wild-type, $\Delta h f q$ and rne mutant strains. RNA was extracted from strains MA3409 (wt), MA7791 $(\Delta h f q)$, and MA9816 (rne-3071). In the experiment in $D$, cells were incubated at $43^{\circ} \mathrm{C}$ for 15 min prior to RNA extraction. RNA was separated on a $1.3 \%$ agarose-formaldehyde gel (top panels) or on an $8 \%$ polyacrylamide/8 $\mathrm{M}$ urea gel (middle and bottom panels). Blots were hybridized with oligonucleotides pp977 (chiPQ, chiP), ppB7 (ChiX), and ppB10 (5S). The rne-3071 allele was constructed by site-directed mutagenesis as described in the Supplemental Material.

A strain carrying chiP::MudK is phenotypically $\mathrm{Lac}^{-}$ and becomes $\mathrm{Lac}^{+}$when Hfq is inactivated. We reasoned that mutations affecting other cell components involved in chiP silencing might similarly result in $\mathrm{a} \mathrm{Lac}^{+}$phenotype. Such mutants were sought using growth on lactose as positive selection. The $\mathrm{Lac}^{+}$mutations mapped at three different loci: (1) the $h f q$ gene; (2) the chiP::MudK locus, and (3) the gene encoding the Salmonella ortholog of SroB (RybC) sRNA (Vogel et al. 2003; Zhang et al. 2003). The latter findings suggested that SroB, renamed ChiX, might participate together with $\mathrm{Hfq}$ in chiP down-regulation.

ChiX sRNA is readily detectable in Northern blots of RNA from wild-type cells (Fig. 1C). In contrast, the sRNA is not detected in cells lacking Hfq. Disappearance of ChiX in the $h f q$ mutant correlates with the appearance of two chiP-related RNAs: a longer species extending to the end of the $y b f N$ gene (renamed $c h i Q$ ) downstream from chiP (Fig. 1A), and a shorter species terminating within the intercistronic region (ICR) between chiP and chiQ. The same two species accumulate in a RNase E temperaturesensitive (ts) mutant incubated at the restrictive temperature (Fig. 1D). Taken together, these data allow us 
to infer that normally, ChiX activity leads to RNase E-dependent degradation of chiP transcripts.

\section{ChiP is an inducible chitoporin}

In an attempt to identify additional players involved in ChiP regulation, we searched for mutations conferring a conditional $\mathrm{Lac}^{+}$phenotype. The lac fusion used in these experiments, chiP91::pCE40(lac), carries the $1 a c Z$ coding sequence of plasmid pCE40 (Ellermeier et al. 2002) joined to the 9 th codon of chiP. This construct expresses $l a c Z$ at much higher levels than the original chiP::MudK fusion. Mutants selected at $30^{\circ} \mathrm{C}$ were screened for the presence of isolates that did not form colonies on lactose plates at $42^{\circ} \mathrm{C}$. Two such mutants were identified. Conceivably, they carried ts alleles of a factor involved in the regulation of $c h i P$, or $c h i X$, or both. Genetic mapping and DNA sequence analysis identified this factor as ChbR, the AraC-type activator of the chitobiose operon, chb (Keyhani and Roseman 1997). The chb operon (chbBCARFG) includes genes for transport and utilization of chitobiose, along with the $c h b R$ regulatory gene (Keyhani and Roseman 1997; Plumbridge and Pellegrini 2004). Chitobiose (the dimer of $\mathrm{N}$-acetylglucosamine) and other chitooligosaccharides (e.g., chitotriose) are produced by the enzymatic breakdown of chitin, the main component of the exoskeleton of arthropods. The involvement of ChbR in chiP regulation, combined with the outer membrane localization of the ChiP protein, suggested that the latter was a porin dedicated to the uptake of chitin-derived oligosaccharides. Consistent with this hypothesis, we observed that both chitobiose and chitotriose effectively induce chiP mRNA synthesis as well as ChiP protein accumulation in the outer membrane (Fig. 2A,B). Furthermore, the chiP gene is absolutely required for Salmonella to be able to grow on chitotriose as the sole source for both carbon and nitrogen (Fig. 2C). A similar requirement is observed with chitobiose, but only at low concentrations, suggesting the existence on an additional, lower affinity uptake system for the disaccharide (data not shown). Existence of this system could explain why chitobiose, but not chitotriose, induces expression of the chiP-lac fusion in spite of chiP being disrupted by the lac sequence (Fig. 2D). In contrast, both sugars are equally efficient at inducing expression of a chiQ-lac fusion, provided that chiP is functional (Fig. $2 \mathrm{E})$. The latter data indicate that chiQ and chiP genes are coordinately regulated. ChiQ, however, appears dispensable for growth on either chitobiose or chitotriose (Fig. $2 \mathrm{C}_{\text {; }}$ data not shown).

In $c h i X^{+}$strains, chiPQ levels are negligible in the absence of inducers and rise $>200$-fold upon induction (Fig. 2A-C). In contrast, in strains deleted for the chiX gene, chitobiose exposure causes chiPQ expression to increase approximately fourfold over an already high basal level (Fig. 2D). The increase depends on the ChbR protein, and may reflect a direct stimulation of chiPQ transcription. Thus, ChiX tightens the response and amplifies the regulatory range. Failure of chitobiose or chitotriose to induce the chiPQ operon in a strain in which the $c h b R$ gene is disrupted by a transposon insertion (Fig. 2D) confirms the ChbR requirement for induction. ChbR is thought to undergo an allosteric change upon binding to a chitobiose-derived metabolite
Figure 2. Regulation of the chiPQ Operon. (A) Northern blot analysis of chiPQ RNA. Cultures from strains MA3409 (wt), MA8933 ( $\Delta$ chiX), and MA9816 (rne-3071) were grown in LB or in LB supplemented with chitobiose $\left(2 \mathrm{mM}\right.$; added at an $\mathrm{OD}_{600}$ of 0.2 ) to an $\mathrm{OD}_{600}$ of 0.35 . RNA was extracted and analyzed as described in the legend to Figure 1C. MA9816 cultures were shifted to $43^{\circ} \mathrm{C} 15$ min prior to RNA extraction. RNA blots were hybridized to ${ }^{32} \mathrm{P}$-labeled oligonucleotides ppB68 (chiPQ and chiP) and pp813 (ssr $A) .(B)$ OMP patterns in the absence and presence of chitotriose. Salmonella OMPs were prepared from $2 \mathrm{~mL}$ cultures of strains MA3409 (wt) and MA8933 ( $\Delta$ chiX) grown overnight in LB without or with $2 \mathrm{mM}$ chitotriose. (C) Genetic requirements for Salmonella growth on chitotriose. Cultures from strains MA3409 (wt), MA9131 (chiP::scar), MA9654 (chiQ::scar), and MA9843 (chbR:: Tn5-TPOP) were grown overnight in NCE medium supplemented with $0.2 \%$ glycerol were spotted on an NCN plate containing 2 mM chitotriose. Strains MA9131 and MA9654 carry small deletions in the $5^{\prime}$-end portions of chiP and chiQ genes, respectively. $(D)$ Expression of chiP91-lacZ fusion. Cultures were grown overnight in NCE $0.2 \%$ glycerol medium without or with $2 \mathrm{mM}$ chitobiose or $2 \mathrm{mM}$ chitotriose added. $\beta$-Galactosidase activity was measured in strains MA9132 (chiPlacZ), MA9184 (chiP-lacZ $\Delta$ chiX), MA9844 (chiP-lacZ chbR::Tn5-TPOP), and MA10049 (chiP-lacZ $\Delta$ chiX chbR::Tn5-TPOP). (E) Expression of chiQ92-lacZ fusion. Strains used were MA9655 (chiQ-lacZ), MA9841 (chiQ-lacZ $\Delta$ chiX), and MA9923 ( $\Delta$ chiP chiQ-lacZ). 
(Plumbridge and Pellegrini 2004; Kachroo et al. 2007). Conceivably, the two conditional ChbR alleles described above (S135L and N238Y) may mimic the allosteric change at low temperature in the absence of inducers.

\section{Mutational analysis of ChiX sRNA}

The Lac-based screen proved suitable to a genetic dissection of ChiX sRNA function. $\mathrm{Lac}^{+}$mutants arising spontaneously, or generated by mutagenizing the chiX gene with the PCR, were isolated from the strain carrying the chromosomal chiP::MudK(lac) insert. The effects of mutations on chiP expression were quantified measuring chiPQ mRNA in Northern blots and assaying $\beta$-galactosidase activity in strains with the chiP91:: pCE40(lac) fusion.

Several chiX mutations affected a 12-base segment complementary to a sequence spanning the ShineDalgarno motif of the chiP gene (Fig. 3A). This suggests a base pair interaction to be responsible for ChiX-mediated repression. Most of the changes relieve repression completely, while some have less pronounced effects (Fig.
3B,C). The differences can be ascribed to the nature of the change (i.e., A51G replaces a U:A base pair by a U:G pair) or to its position in the duplex. Mismatches in the middle of the segment (U47C, U49C) appear less detrimental to regulatory proficiency than the ones on the sides (e.g., U45C). Mutants with a weaker phenotype express significant LacZ activity and yet show little or no chiPQ RNA in the Northern blots (Fig. 3, cf. B and C). This discrepancy could originate from RNA cleavage events occurring in between rounds of translation initiation. Even though some ribosomes can reach the end of the message (yielding a protein product and measurable LacZ activity), no chiP RNA molecule ever exists in its full length. Northern blot analysis of processing intermediates from the 3' end of the chiQ gene supports this interpretation (Supplemental Fig. S2).

Two chiP mutations obtained in the initial $\mathrm{Lac}^{+}$selection fall within the presumptive ChiX pairing window (Fig. 3A). The change in chiP C74A is predicted to restore base-pairing if combined with ChiX G50U. Results in Figure 3D show that chiP C74A disrupts regulation when present alone but not in combination with the ChiX

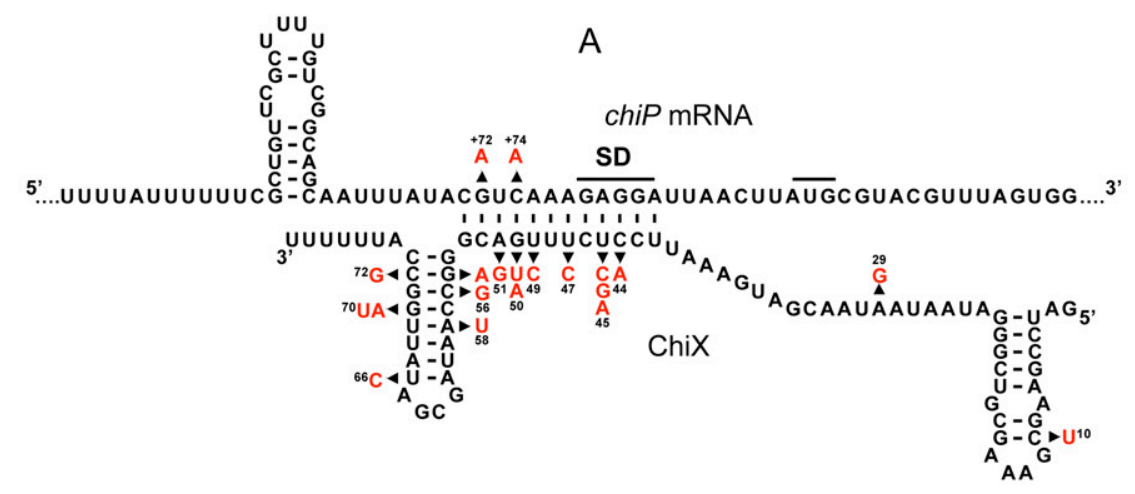

B
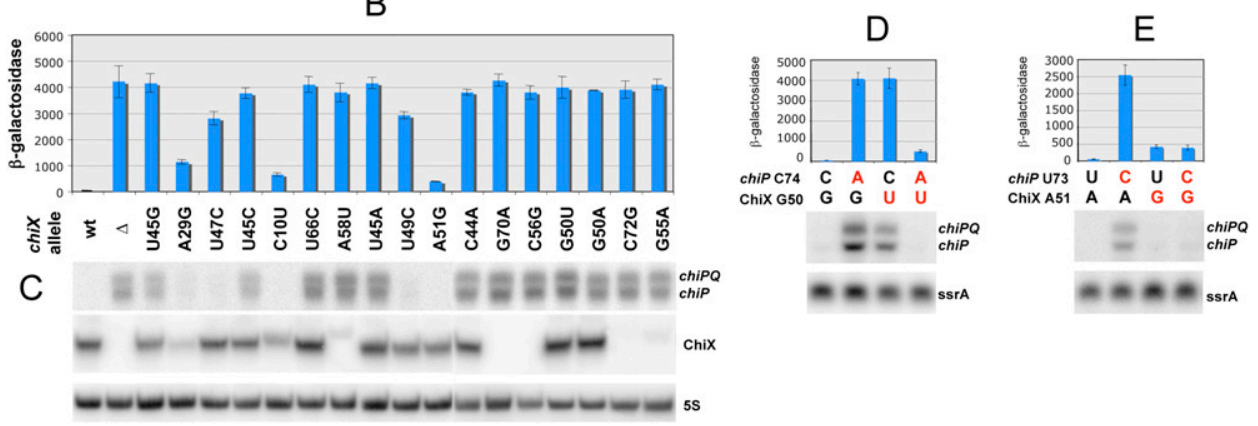

Figure 3. Analysis of ChiX sRNA mutations relieving chiP silencing. Mutants were all derived from strain MA8110 (chiP::MudK) either selecting for growth on NCE-minimal lactose plate (spontaneous Lac ${ }^{+}$mutants) or by PCR mutagenesis of the chiX gene. In this second procedure, mutants were identified as blue colonies on LB plates supplemented with X-gal. Due to the sensitivity of the colorimetric screen, the latter method yielded some alleles causing more subtle alterations. Following isolation, the chiX mutations were moved into the chiP91::pCE40(1ac) background (strain MA9132). (A) Model for the pairing interaction between chiP mRNA 5' UTR and ChiX sRNA. The base changes conferring a Lac ${ }^{+}$phenotype are indicated. The $5^{\prime}$ ends of chiP and chiX transcripts were determined by primer extension (Supplemental Fig. S1). (B) Effect of chiX mutations on expression of the chiP91-lacZ fusion. $\beta$-Galactosidase activity was measured on cultures grown overnight in LB medium $\left(\mathrm{OD}_{600} \approx 3\right)$. (C) Northern blot analysis of RNA from chiX mutants. RNA was extracted and analyzed as in Figure 2A. $(D, E)$. Effects of compensatory changes in the chiP/ChiX RNA duplex on chiP expression. The strains used in $B$ and $C$ are listed in Supplemental Table S3, strains used in $D$ and $E$ are listed in Supplemental Table S4. 
G50U allele. Similar compensatory effects were observed with other chiP changes obtained by site-directed mutagenesis (Fig. 3E; data not shown). These data provide evidence that repression by ChiX sRNA involves basepairing with chiP mRNA. Likely, formation of the RNA duplex inhibits chiP translation initiation by interfering with ribosome binding.

In chiX mutants carrying changes outside the pairing interval, altered $c h i P$ regulation correlates with a decrease in ChiX sRNA levels (Fig. 3A-C). Most of these mutations disrupt the Rho-independent terminator hairpin, a structure thought to be important for stability in prokaryotic RNAs (Mott et al. 1985; Aiba et al. 1991; Guarneros and Portier 1991). Therefore, the depletion of ChiX sRNA in these mutants is likely due to increased decay. Two alleles with a weaker phenotype, C10U and A29G, are located in the $5^{\prime}$ half of the molecule. We envisaged that these mutations might affect ChiX-Hfq binding. This idea was tested in vitro comparing the affinities of wild-type and mutant sRNAs for purified Hfq in a gel retardation assay. Results confirmed that both C10U and A29G impair the sRNA's ability to bind Hfq (Fig. 4A). Allele A29G falls within an AU-rich sequence (AAUAAUAAUA), which includes two overlapping AAYAA motifs proposed to play a role in $\mathrm{Hfq}$ recognition (Soper and Woodson 2008). Given that the mutation disrupts both repeats (AAUGAUAAUA), the data in Figure 4A further substantiate the role of this sequence in Hfq-RNA interactions.

The C10U mutation causes the replacement of a G:C base pair by a $\mathrm{G}: \mathrm{U}$ base pair in a putative secondary structure at the 5' end of ChiX sRNA (Fig. 3A). Structural analysis (Fig. 4B) reveals that the mutation affects the reactivity of surrounding bases as well as the spacing of bands in the sequence ladder (cf. $\mathrm{OH}^{-}$lanes in Fig. 4B). These results might indicate that the C10U change destabilizes the top portion of the hairpin structure, causing the expansion of the apical loop. Such a perturbation might interfere with the binding of Hfq protein to the adjacent recognition site.

\section{ChiX sRNA is destabilized during chiPQ induction}

Interestingly, ChiX sRNA levels are drastically reduced in cells growing in the presence of chitobiose (Fig. 5A). Considering that this decrease could be the basis of chiP regulation, we set out to investigate the underlying mechanism. Our initial hypothesis-that activated ChbR protein acted as a repressor at the chiX promoter-was ruled out by the analysis of a lacZ gene fusion to the chiX promoter, which showed lacZ expression to remain unchanged in the presence of chitobiose (data not shown). We then postulated that the accumulation of chiPQ mRNA during induction might be sufficient to titrate out ChiX sRNA and stimulate its decay (Massé et al. 2003). However, ChiX depletion was still observed in a strain where $c h i P Q$ transcription cannot take place due to a promoter deletion (Fig. 5A). Nonetheless, the above line of reasoning led us to test whether the ChiX depletion was in some way related to the sRNA's ability to base-pair. Analysis of two of the pairing mutants described earlier (G50A, U45A) revealed ChiX sRNA levels to be significantly less affected in the presence of the G50A allele (Fig. 5A). This suggested that ChiX sRNA undergoes degradation during induction as a result of its participation in a base pair interaction. Having ruled out chiP mRNA involvement, we searched elsewhere for an alternative pairing target. We discovered that the ICR
Figure 4. Characterization of chiX alleles A29G and C10U. Wild-type and mutant ChiX RNA were synthesized in vitro and labeled at their $5^{\prime}$ with ${ }^{32} \mathrm{P}$. (A) Gel shift assay with purified Hfq protein. ${ }^{32}$ P-labeled RNA (4 nM) was incubated at $30^{\circ} \mathrm{C}$ for 30 min with increasing amounts of purified Salmonella Hfq protein $(0,2,11,55,276,1380 \mathrm{nM})$ in $50 \mathrm{mM}$ Tris- $\mathrm{HCl}(\mathrm{pH} 7.8), 50 \mathrm{mM} \mathrm{NaCl}, 50 \mathrm{mM}$ $\mathrm{KCl}, 10 \mathrm{mM} \mathrm{MgCl}$, and $6 \mu \mathrm{M}$ yeast tRNA. Samples were loaded on a $5 \%$ nondenaturing polyacrylamide gel and radioactivity detected and quantified by phosphorimaging. (B). Structural analysis of wild-type and mutant RNAs. 5'-End-labeled RNAs were subjected to the indicated treatments followed by separation on a denaturating polyacrylamide gel. (C) Untreated control; $(\mathrm{OH})$ alkaline ladder. Note the effect of the C10U mutation on band spacing in the $\mathrm{OH}$ lane as well as in the RNase A-treated samples (e.g., U25).
A

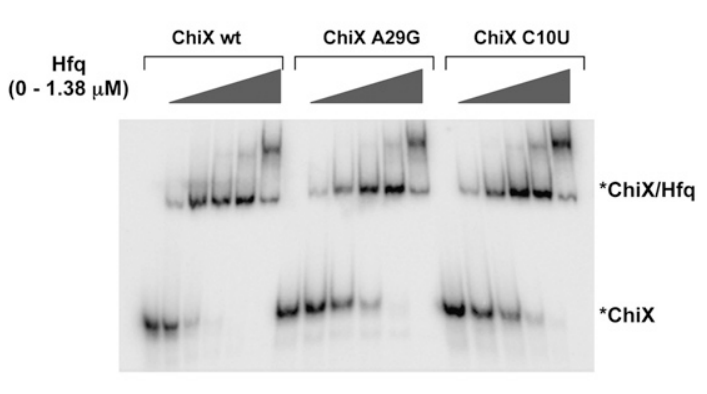

B

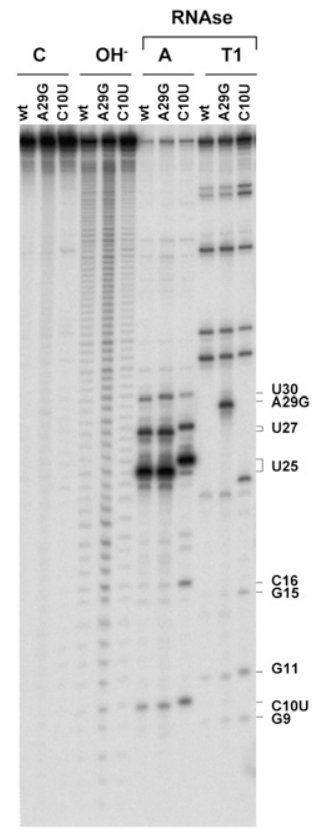


A
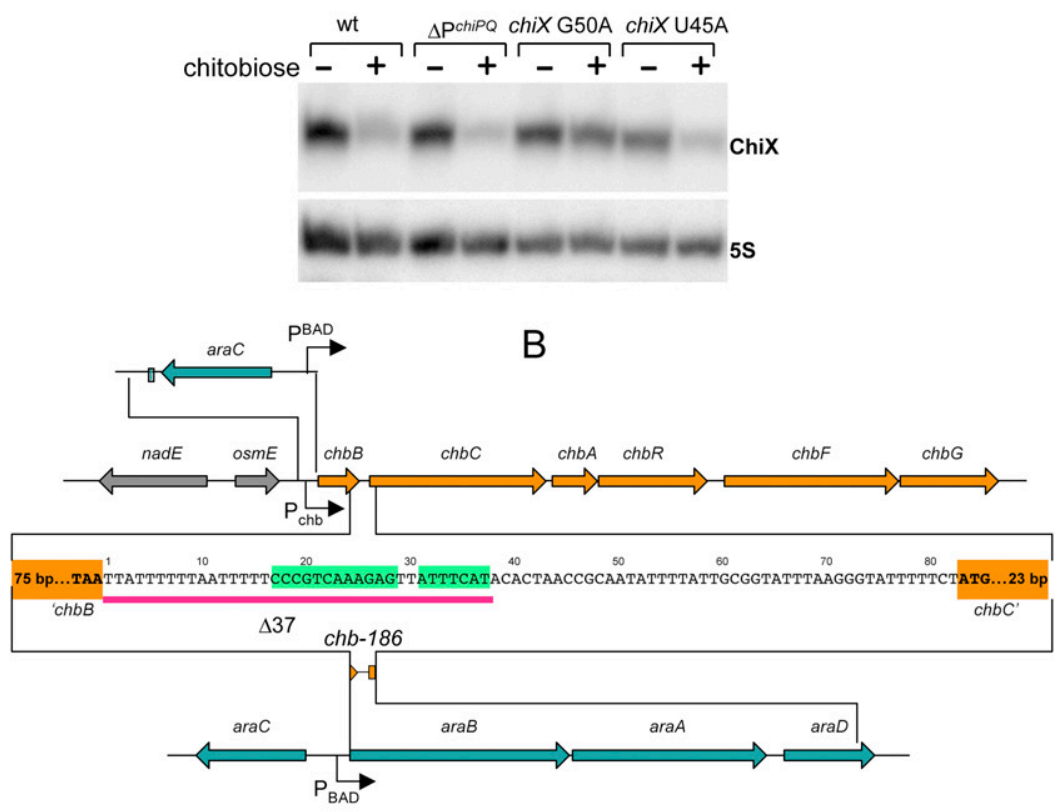

C

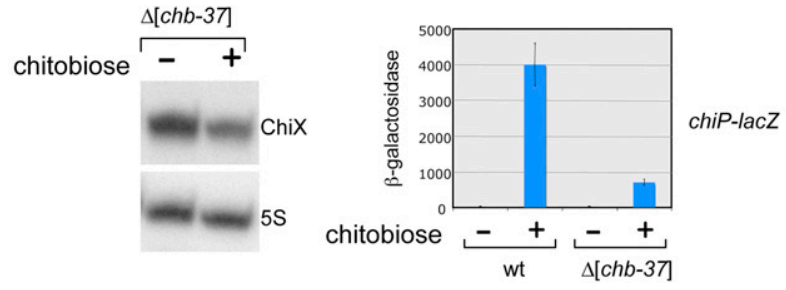

Figure 5. Involvement of the chb operon in chiP regulation. (A) Northern blot analysis of ChiX sRNA. Cultures from strains MA3409 (wt), MA9924 ( $\left.\Delta \mathrm{P}^{\text {chiPQ}}\right)$, MA9010 (chiX G50A), MA9267 (chiX U45A) were grown in NCE $0.2 \%$ glycerol medium without or with $2 \mathrm{mM}$ chitobiose to an $\mathrm{OD}_{600}$ of 0.35 . RNA was extracted, separated on an $8 \%$ polyacrylamide/8 $\mathrm{M}$ urea gel, and transferred to Hybond- $\mathrm{N}^{+}$membranes. Blots were hybridized to ${ }^{32} \mathrm{P}$-labeled oligonucleotides ppB7 (ChiX), ppB10 (5S). (B) Schematic diagram of the Salmonella chb operon and its ara operon fused derivatives. The ICR between $c h b B$ and $c h b C$ genes (sense strand) is enlarged, with the ChiX complementary segment boxed in green. The red bar underlining a portion of the chbBC ICR indicates the 37-bp deletion analyzed in this study. A 186-bp segment spanning the entire chbBC ICR (chb-186) was inserted in place of the structural portion of the ara operon. $(C)$ Northern blot analysis of ChiX sRNA from the chb 37 mutant. RNA was extracted from strain MA10004 (chbs37) and processed as in $A .(D)$ Effect of $c h b \Delta 37$ on the regulation of the chiP91lac fusion. $\beta$-Galactosidase activity was measured in strain MA10074 ( $\Delta$ chb-37 chiP-lac) as described in Figure 2D. between the first two genes of the $c h b$ operon, $c h b B$ and $c h b C$, encodes a sequence complementary to most of the pairing segment of ChiX sRNA (Fig. 5B). These findings were all the more interesting as the $c h b$ RNA is expected to accumulate in the presence of chitobiose, and thus it could easily become in excess of ChiX sRNA.

\section{Transcription of chbBC ICR leads to ChiX sRNA destabilization}

A 37-base-pair (bp) segment covering the chbBC ICR portion complementary to ChiX (Fig. 5B) was deleted by replacement with the 75-bp "scar" sequence from plasmid pKD13 (Datsenko and Wanner 2000). The deleted strain still suffered a ChiX sRNA decrease when exposed to chitobiose; however, the variation was significantly less than in wild type (Fig. 5C). Possibly, some ChiX sRNA decay occurs as a result of pairing with chiP mRNA, whose levels increase due to ChbR-mediated transcriptional activation (see above). As one might expect, the $c h b \Delta 37$ deletion prevents full induction of the chiP-lac fusion (Fig. 5D). Residual induction can be ascribed to the increase in chiP mRNA levels saturating the inhibitory capacity of ChiX sRNA. Similar ChiX titration effects were observed upon raising chiP mRNA levels artificially, as in a strain with a chromosomal $\mathrm{P}^{\mathrm{BAD}}$. chiP fusion grown in the presence of arabinose or in a chiP "promoter-UP" mutant isolated in the Lac selection above (data not shown).

The $c h b$ promoter was replaced by a module comprising the araC gene and the $\mathrm{P}^{\mathrm{BAD}}$ promoter (Fig. 5B). Analysis of the resulting strain showed ChiX sRNA levels to sharply decline when cells were grown in the presence of arabinose (Fig. 6A). In contrast, a similar construct with the $\mathrm{P}^{\mathrm{BAD}}$ promoter controlling chiPQ transcription had only a modest effect on ChiX sRNA levels (Fig. 6A). Finally, a 186-bp DNA fragment spanning the chbBC ICR was translocated into the ara operon, placed under the control of the $\mathrm{P}^{\mathrm{BAD}}$ promoter (Fig. 5B). The resulting strain reproduced the effect of arabinose on ChiX sRNA levels (Fig. 6A), confirming the $c h b B C$ ICR to be solely responsible for ChiX sRNA decay.

As expected, arabinose induction of the $\mathrm{P}^{\mathrm{BAD}}-c h b$ fusion constructs leads to chiPQ derepression. However, the chiPQ increase is significantly less dramatic than normally observed following chitobiose exposure. Ascribing this difference to the lack of ChbR activation, the induction experiments were repeated in the presence of 

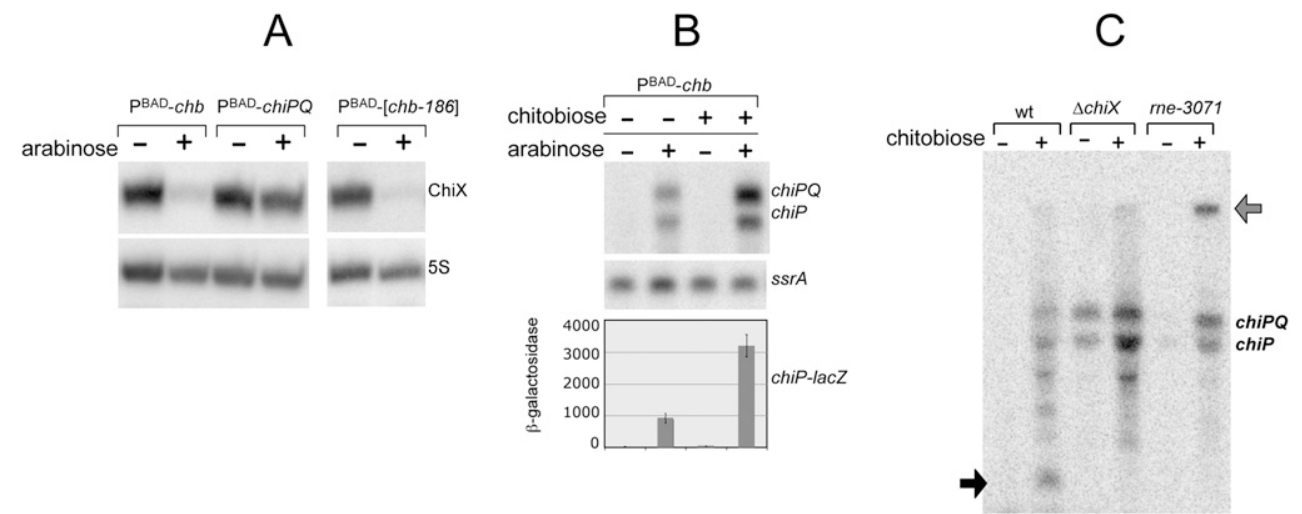

Figure 6. Role of $c h b B C$ ICR in ChiX sRNA turnover. $(A)$ Effect of $c h b B C$ ICR transcription on ChiX sRNA levels. RNA was extracted from cells growing in NCE $0.2 \%$ glycerol without or with chitobiose $(2 \mathrm{mM})$ and/or $0.02 \%$ arabinose at an $\mathrm{OD}_{600}$ of 0.35 and processed

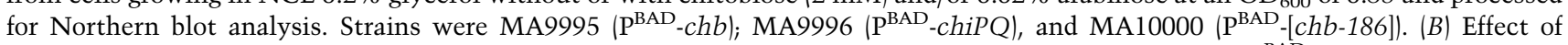
$c h b B C$ ICR transcription on $c h i P$ expression. Northern blot analysis was carried out with strain MA9995 (P ${ }^{\mathrm{BAD}}-$ chb). $\beta$-Galactosidase activity was measured in strain MA10031 ( $\mathrm{P}^{\mathrm{BAD}}-$ chb chiP-lac). (C) Northern blot analysis of chb RNA. The Northern blot in Fig. 2A was hybridized to a ${ }^{32} \mathrm{P}$-labeled oligonucleotide from the $c h b B C$ ICR (ppD96) without stripping from previous chiPQ probe.

chitobiose (in the strain carrying the entire chb operon fused to the $\mathrm{P}^{\mathrm{BAD}}$ promoter). Results confirmed that maximal chiPQ derepression is attained when both sugars are concomitantly present (Fig. 6B). These data further support the idea that ChiP induction normally results from coupling ChiX sRNA decay to the stimulation of chiP transcription.

To gather information on the chbBC ICR transcript, the RNA blot in Figure 2A was rehybridized to a probe covering the segment pairing to ChiX. This analysis detected an $\sim 300$-base RNA species accumulating during chitobiose induction (solid arrow in Fig. 6C). Interestingly, this species is not present in the chiX deletion mutant, suggesting that its formation is consequent to pairing with ChiX sRNA. Failure to detect the 300-base RNA in the rne ts mutant, together with the accumulation of a high molecular RNA band in this strain (open arrow in Fig. 6C), points to the involvement of RNase $\mathrm{E}$ in the pathway leading to the 300-base RNA.

\section{The chbBC ICR RNA invades ChiX sRNA transcription terminator}

The data in Figure 6A suggest that ChiX sRNA is far more susceptible to degradation when pairing with the $c h b B C$ ICR than with the chiP 5' UTR. A peculiarity of the $\operatorname{chbBC}$ spacer element is that the region of complementarity to ChiX sRNA extends two nucleotides into the transcription terminator hairpin of the sRNA (Fig. 7A). This raises the possibility that the pairing interaction with $c h b B C$ ICR RNA disrupts the bottom portion of ChiX terminator stem, causing the stem to become $2 \mathrm{bp}$ shorter. Given the importance of this region for the overall stability of ChiX sRNA (see mutant analysis above) one expects that the loss of $2 \mathrm{bp}$ would dramatically accelerate sRNA decay. The hypothesis predicts that affecting the ability of the $c h b B C$ sequence to basepair with the terminator portion should prevent its degradation. This prediction was tested by changing either of the two $\mathrm{C}$ residues involved to $\mathrm{G}$ residues. Results in Figure 7B show that either change attenuates ChiX sRNA decay following activation of $c h b B C$ ICR transcription. Concomitantly, either mutation completely abolishes chiP derepression (Fig. 7B). Therefore, these observations confirm that the first 2 bases on the $5^{\prime}$ side of ChiX attenuator stem participate in $c h b B C$ ICR pairing. Shortening of the stem might make ChiX sRNA more susceptible to degradation.

\section{Discussion}

In this study, we describe the existence of a chitoporin (ChiP) in Salmonella, as well as the mechanism by which synthesis of this protein is regulated as a function of substrate availability. ChiP synthesis is induced in the presence of chitooligosaccharides as a result of two combined events: stimulation of chiP gene transcription and relief of chiP translational repression by ChiX small RNA. Both events are triggered by the transcriptional activation of the $c h b$ operon, which encodes the components for chitobiose (and chitotriose) phosphoenolpyruvate-dependent phosphotransferase system (PTS) along with the ChbR regulator protein (Plumbridge and Pellegrini 2004). Binding of a chitobiose/chitotriose-derived metabolite to ChbR causes this protein to activate chb transcription, thus increasing ChbR levels. Inducer-bound ChbR stimulates chiP gene transcription. Concomitantly, an RNA sequence processed from the polycistronic chb transcript (the chbBC ICR element) targets ChiX sRNA for degradation. Altogether, these coordinate events may allow coupling ChiP-mediated uptake of chitooligosaccharides to their transport across the inner membrane (Fig. 8).

The mechanism by which the $c h b B C$ ICR RNA promotes the destruction of ChiX sRNA is unprecedented. ChiX degradation appears to result from $c h b B C$ ICR RNA pairing with the portion of the sRNA that makes up the 

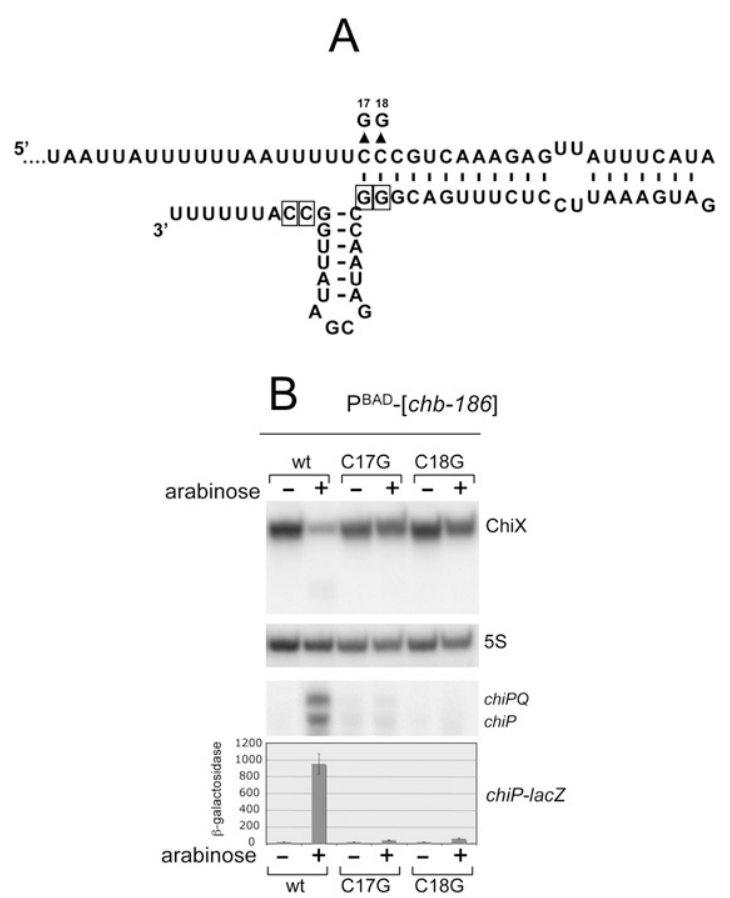

Figure 7. Analysis of mutations affecting pairing of $c h b B C$ ICR RNA with bases in ChiX sRNA transcription terminator stem. (A) Model for $c h b B C$ ICR pairing to ChiX sRNA. chbBC ICR bases are numbered starting to the right of the translation stop codon (UAA) of the $c h b B$ gene. $(B)$ Analysis of the effects of the mutations on ChiX sRNA levels and on chiPQ regulation. RNA and $\beta$-galactosidase measurements were carried out under the same conditions as in Figure 6. Strains used in the Northern blot analysis of ChiX sRNA (top and middle panels) and for the $\beta$-galactosidase determinations were MA10043 ( $\mathrm{P}^{\mathrm{BAD}}{ }_{-}[$chb-186] chiP-lac), MA10044 (P $\mathrm{P}^{\mathrm{BAD}}$-[chb-186 C17G] chiP-lac), and MA10045 ( $\mathrm{P}^{\mathrm{BAD}}$-[chb-186 C18G] chiP-lac). Strains used for Northern blot analysis of chiPQ mRNA were MA10051 ( $\mathrm{P}^{\mathrm{BAD}}-[$ chb-186]),

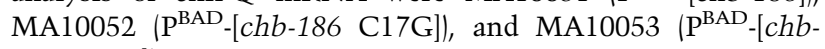
$186 \mathrm{C} 18 \mathrm{G}])$.

Rho-independent transcription terminator. This suggests that degradation is caused by disruption/destabilization of the terminator stem-loop structure. Thus, the action of $c h b B C$ ICR RNA would be equivalent to the effect of mutations that destabilize the terminator, a number of which were described here. Similar mutations in a phage $\lambda$ terminator were shown to make the RNA transcript susceptible to $3^{\prime}$ exonucleolytic degradation (Cisneros et al. 1996). It seems possible that a $3^{\prime}$ exonuclease could intervene in the $\operatorname{chbBC}$ ICR-dependent degradation of ChiX sRNA.

We found a 300-base RNA containing the $c h b B C$ ICR sequence to accumulate as a result of pairing with ChiX sRNA. Whether this RNA species is produced by a processing pathway specifically activated by the formation of the duplex, or simply reflects the stabilization of a short-lived intermediate remains to be determined. In either case, the accumulation of the presumptive chb primary transcript in an RNase E mutant leaves little doubt that this activity is responsible for the initial cleavage events. An implication of the rapid turnover is that the regulatory action of $c h b B C$ ICR element must depend on continuous $c h b$ operon transcription. Conceivably, this allows the system to quickly readjust once the inducer concentration falls and, with it, the requirement for ChiP activity. One might wonder about what makes such a complex transcriptional-translation control to be preferred to a more classical regulation mediated by the ChbR activator protein only. Particularly counterintuitive in the current mechanism is that chiP repression under uninduced conditions relies on both chiP and chiX genes to be continuously transcribed. Perhaps the answer lies in the timing of the induction response. Having the chiP mRNA already present when ChiX sRNA concentration begins to fall (concomitant with the appearance of the chbBC ICR RNA) might allow ChiP synthesis to start before the chb operon is fully induced; namely, before accumulation of the ChbR activator. The ChiX component of the regulatory mechanism could therefore allow for more efficient priming of the induction switch.

The ChiP protein is highly conserved in enteric bacteria with sequence identities exceeding $90 \%$ in the Escherichia and Shigella orthologs. The protein belongs to the OprD family and is phylogenetically unrelated to the chitoporin from Vibrio species (Keyhani et al. 2000). Chitin is the most commonly occurring nitrogencontaining polysaccharide in nature. Since Salmonella and E. coli do not naturally secrete chitinases (Francetic et al. 2000), these organisms probably rely on other microorganisms producing the ChiP substrates in the environment. This could occur through the activity of other, chitinase-proficient microorganisms or metabolic processes in eucaryotic hosts. It is relevant in this respect that an increasing number of chitinases have been identified recently in a variety of mammalian species including humans (Escott and Adams 1995; Zhu et al. 2004).

After this work was completed, a report has appeared describing the silencing of ChiP (YbfM) by ChiX (named MicM) in E. coli (Rasmussen et al. 2009). Rasmussen et al. (2009) presented evidence for the base-pairing interaction between the sRNA and the chiP 5' UTR and substantiated the importance of $\mathrm{Hfq}$ as a catalyst of the interaction. However, the study did not elucidate the function of ChiP nor the basis for ChiX (MicM)-dependent regulation. At the same time, a separate report identified ChiX (named $\mathrm{RybC}$ ) as an sRNA down-regulating the $d p i B$ gene for the sensor component of a two-component system (DpiA/B) required for citrate fermentation and linked to various phenotypes including SOS induction and plasmid stability in E. coli (Mandin and Gottesman 2009). While the role of ChiX in any of these phenotypes remains elusive, the presence of citrate carrier/utilization genes, citA/ citB, immediately adjacent to the chiPQ operon in Salmonella (see Fig. 1A) may suggest a link between ChiP and citrate metabolism. (Note that no citA/citB orthologs exist in the E. coli, where, somewhat confusingly, CitA/B is an alternative designation for the DpiA/B system) (Kaspar and Bott 2002). We notice that pairing of $d p i B 5^{\prime}$ UTR with ChiX RNA extends 1 base $(2$ bases allowing a G:U pair) into the ChiX transcription terminator (Mandin and Gottesman 2009). This raises the 
Figueroa-Bossi et al.

Figure 8. Model for chiP regulation. $(A)$ In the absence of inducers, the chiX gene (black arrow) and the chiPQ operon (green arrows) are transcribed constitutively. Pairing of ChiX sRNA with the $5^{\prime}$ UTR of chiPQ mRNA inhibits ChiP synthesis, promoting cleavage of the chiP mRNA by RNase E. The chb operon (blue arrows; only three genes shown) is expressed just to the level needed to prime the system. (B) In the presence of chitooligosaccharides, inducer binding to ChbR causes this protein to activate transcription of the $c h b$ operon and to further stimulate chiPQ transcription. Processing of the $c h b$ transcript (initiated by RNase E) releases chbBC ICR RNA. This RNA base-pairs with ChiX sRNA making it susceptible to the action of a ribonuclease. The drop in ChiX levels, combined with the increase in chiPQ mRNA, relieves chiP repression leading to a burst of ChiP synthesis. ChiP assembles in the outer membrane resulting in more inducer uptake.

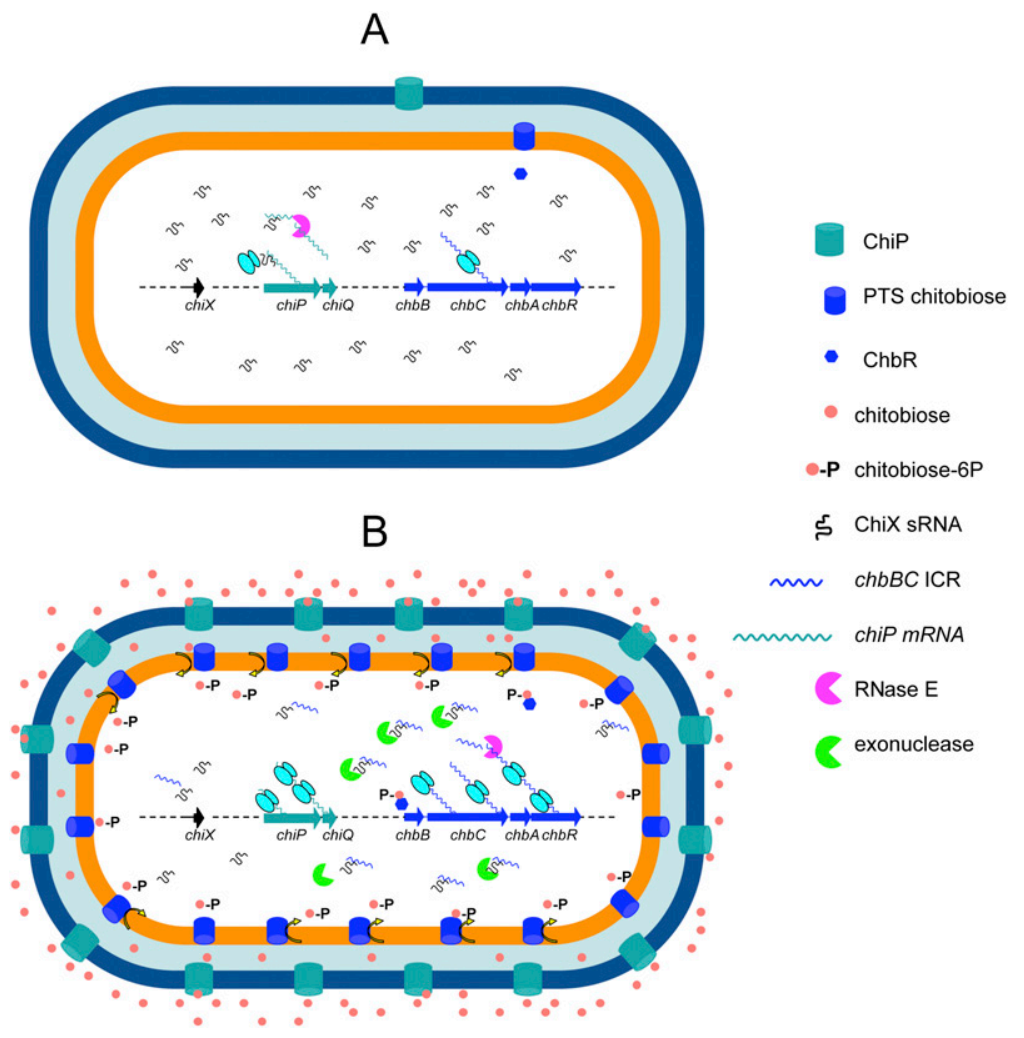

Supplemental Table S1. The construction of these strains is detailed in the Supplemental Material. Bacteria were cultured at $37^{\circ} \mathrm{C}$ in liquid media or in media solidified by the addition of $1.5 \%$ Difco agar. LB broth (Bertani 2004) was used as complex medium. Carbon-free medium (NCE) and carbon-free and nitrogen-free medium (NCN) (Maloy and Roth 1983), supplemented with the appropriate carbon and/or nitrogen sources, were used as minimal media. Typically NCE medium was supplemented with $0.2 \%$ glycerol. Antibiotics (Sigma) were included at the following final concentrations (in LB): chloramphenicol, $10 \mu \mathrm{g} \mathrm{mL}^{-1}$; kanamycin monosulphate, $50 \mu \mathrm{g} \mathrm{mL}^{-1}$; sodium ampicillin, $75 \mu \mathrm{g} \mathrm{mL}^{-1}$; spectinomycin dihydrochloride, $80 \mu \mathrm{g}$ $\mathrm{mL}^{-1}$; tetracycline hydrochloride, $25 \mu \mathrm{g} \mathrm{mL}^{-1}$. LB plates containing 5-bromo-4-chloro-3-indolyl- $\beta$-D-galactopyranoside (X-gal; from Sigma), $40 \mu \mathrm{g} \mathrm{mL}^{-1}$ were used to monitor lacZ expression in bacterial colonies. Liquid cultures were grown in New Brunswick gyrotory shakers and growth was monitored by measuring the optical density at $600 \mathrm{~nm}$ with a Milton-Roy Spectronic 301 spectrophotometer.

\section{Enzymes and chemicals}

Restriction enzymes, T4 polynucleotide kinase and Taq DNA polymerase were from New England Biolabs, Pfu-Turbo DNA polymerase was from Stratagene, T4 DNA ligase was from USB. DNA oligonucleotides were obtained from Sigma Aldrich. A list with all oligonucleotides used in this work is in Supplemental Table S2. Acrylamide-bisacrylamide $(30 \%, 29: 1)$ and other electrophoresis reagents were from Bio-Rad. Agarose was from Invitrogen. Hybond- $\mathrm{N}^{+}$membranes and hybridization buffer used for Northern blot analysis were from GE Healthcare and from Applied Biosystems-Ambion, respectively. MegashortScript T7 kit, Kinase Max kit, RNases A and T1, yeast tRNA were all from Applied Biosystems-Ambion. 


\section{Genetic techniques}

Generalized transduction was perfomed using the highfrequency transducing mutant of phage P22, HT 105/1 int-201 (Schmieger 1972) as described (Lemire et al. 2008). Chromosomal engineering was carried out by the $\lambda$ red recombination method (Datsenko and Wanner 2000; Yu et al. 2000) as implemented by Datsenko and Wanner (2000) and Uzzau et al. (2001).

Donor DNA fragments were generated by PCR using plasmid or chromosomal DNA templates. Preparation of recipient bacteria, DNA electroporation, and isolation and processing of recombinant clones were carried out as described (Lemire et al. 2008).

\section{$\beta$-Galactosidase assays}

$\beta$-Galactosidase activity was measured in toluene-permeabilized cells as described by Miller (1992), and is expressed in Miller units throughout this work.

\section{OMP preparation}

Salmonella OMP fraction was purifed by the method of Santiviago et al. (2003) and analyzed by polyacrylamide gel electrophoresis as described previosuly (Bossi and Figueroa-Bossi 2007).

\section{RNA extraction and Northern analysis}

RNA was prepared by the acid-hot-phenol method from exponentially growing cells $\left(\mathrm{OD}_{600}\right.$ of 0.35$)$ as described previously (Bossi and Figueroa-Bossi 2007). RNA was separated on a 1.3\% agarose-formaldehyde gel or on an $8 \%$ polyacrylamide/8 $\mathrm{M}$ urea gel and transferred to Hybond- $\mathrm{N}^{+}$membranes. Blots were hybridized to DNA oligonucleotides labeled at the $5^{\prime}$ ends with T4 polynucleotide kinase.

\section{In vitro RNA synthesis, purification, and labeling}

A template DNA carrying the chiX gene fused to the T7 promoter was generated by PCR from genomic DNA. To produce wild-type and chiX A29G mutant templates, amplification was with primers ppC19 and ppC20 on genomic DNA from wild-type and from strain MA9220, respectively. For the chiX C10U template, primers were ppC60 (carrying the C10U change) and ppC20, and genomic DNA was from strain MA9226 (see Supplemental Tables S1, S2). In vitro transcription was performed with the MegashortscriptT7 kit (Ambion AM1354) following the manufacturer's protocol. After incubation for $2 \mathrm{~h}$ at $37^{\circ} \mathrm{C}$, DNase was added and incubation continued for further $15 \mathrm{~min}$, followed by phenol extraction and overnight precipitation in ethanol/ sodium acetate/glycogen. RNA was recovered by centrifugation, resuspended in nuclease-free water, and its concentration determined by nanodrop reading. The RNA solution was adjusted to $5 \mathrm{pmol} / \mu \mathrm{L}$. RNA (10 pmol) was dephosphorylated and labeled at its 5 ' end with $\left[\gamma-{ }^{32} \mathrm{P}\right]$ ATP $(3000 \mathrm{Ci} / \mathrm{mmol}$ from Perkin-Elmer) using the KinaseMax kit (Ambion AM1520). Labeled RNA was purified by $8 \%$ PAGE. After extraction from gel, RNA was phenolextracted, ethanol precipitated, and resuspended in nuclease-free water. Before use in binding and structural studies, RNA was heated in refolding buffer $(50 \mathrm{mM}$ Tris at $\mathrm{pH} 8,0.1 \mathrm{M} \mathrm{NaCl}, 0.1 \mathrm{M}$ $\mathrm{KCl}, 1 \mathrm{mM} \mathrm{MgCl}$ ) for $3 \mathrm{~min}$ at $85^{\circ} \mathrm{C}$, followed by $20 \mathrm{~min}$ at room temperature, and finally placed on ice.

\section{Hfq protein purification}

The Salmonella $h f q$ gene was cloned into plasmid pNFB28, a pET-16b (Novagen) derivative designed to obtain either
C-terminal or N-terminal $(7 \times)$ His-tagged fusions to genes of interest. C-terminally tagged Hfq protein was purified from $E$. coli BL21 codon ${ }^{+}$cells carrying the plasmid clone. Bacteria were grown in $1 \mathrm{~L}$ of LB medium supplemented with ampicillin (100 $\mathrm{mg} / \mathrm{L}$ ) at $37^{\circ} \mathrm{C}$ until OD600 of $0.2 ; 1 \mathrm{mM}$ isopropyl 1-thio-Dgalactopyranoside was added and cells cultured for a further $3 \mathrm{~h}$. Bacteria were harvested by centrifugation at $10,000 \mathrm{~g}$ for $20 \mathrm{~min}$ at $4^{\circ} \mathrm{C}$. Pellets were frozen at $-20^{\circ} \mathrm{C}$ for several hours prior to resuspension in $50 \mathrm{~mL}$ of $50 \mathrm{mM}$ Tris- $\mathrm{HCl}(\mathrm{pH} 7.8), 0.3 \mathrm{M} \mathrm{NaCl}$, $10 \%$ Glycerol, $0.1 \%$ Triton X-100, $100 \mu \mathrm{g} / \mathrm{mL}$ lysozyme, and protease inhibitor cocktail from Sigma. Cells were sonicated by 15 rounds of 10 -sec pulses/10-sec intervals, on ice. Lysate was incubated for $10 \mathrm{~min}$ at $75^{\circ} \mathrm{C}$ and centrifuged at $11,800 \mathrm{~g}$ for 40 min. The supernatant was loaded on a 5-mL HiTrap Chelating HP prepacked column from GE Healthcare activated according to the manufacturer's instructions. Column was equilibrated with $25 \mathrm{~mL}$ of $50 \mathrm{mM}$ Tris- $\mathrm{HCl}(\mathrm{pH} 7.5), 0.3 \mathrm{M} \mathrm{NaCl}$, and the protein eluted with the same buffer supplemented with $0.5 \mathrm{M}$ imidazole. Following SDS-PAGE electrophoresis of elution aliquots, protein containing fraction was incubated for $10 \mathrm{~min}$ at $65^{\circ} \mathrm{C}$. Buffer exchange and concentration steps were performed with Centricon 10K Amicon, ultrafiltration unit (Millipore). Protein was kept at $4^{\circ} \mathrm{C}$ in $50 \mathrm{mM}$ Tris- $\mathrm{HCl}(\mathrm{pH} 7.8), 0.3 \mathrm{M}$ $\mathrm{NaCl}, 10 \%$ glycerol, and $0.1 \%$ Triton $\mathrm{X}-100$. The concentration was determined by Bradford assay.

\section{Gel shift assays}

Labeled ChiX RNA (4 $\mathrm{nM})$, was incubated with increasing amounts of purified Salmonella Hfq as indicated, in $50 \mathrm{mM}$ Tris-HCl (pH 7.8), $50 \mathrm{mM} \mathrm{NaCl}, 50 \mathrm{mM} \mathrm{KCl,10} \mathrm{mM} \mathrm{MgCl}$, and $6 \mu \mathrm{M}$ yeast tRNA for $30 \mathrm{~min}$ at $30^{\circ} \mathrm{C}$. Binding reactions were run on a $5 \%$ nondenaturing polyacrylamide gel in $0.5 \times \mathrm{TBE}$ buffer for $3 \mathrm{~h}$ at $4^{\circ} \mathrm{C}$ at constant current of $15 \mathrm{~mA}$. Results were analyzed by phosphorimaging using ImageQuant software.

\section{RNA structural analyses}

End-labeled RNA was refolded as described above. Enzymatic treatments were performed in $10 \mu \mathrm{L}$ of reaction mix containing $0.2 \mathrm{pmol}$ of RNA, $1 \mu \mathrm{g}$ of yeast tRNA, $1 \times$ Structure buffer (Ambion), and $0.01 \mathrm{ng}$ of RNase A (1 mg/mL; Ambion AM 2274) or $0.1 \mathrm{U}$ of RNase T1 (1 U/mL; Ambion AM 2283). Incubation was for $12 \mathrm{~min}$ at $37^{\circ} \mathrm{C}$. Reactions were stopped by addition of $20 \mu \mathrm{L}$ of completed Precipitation/Inactivation buffer from the same manufacturer. Partial alkaline hydrolysis was performed according to Ambion's protocol as follows: $10 \mu \mathrm{L}$ of reaction mix containing 0.2 pmol of RNA, $1 \mu \mathrm{g}$ of yeast tRNA, $1 \times$ Alkaline Hydrolysis buffer, were incubated for $8 \mathrm{~min}$ at $95^{\circ} \mathrm{C}$, placed on ice, and $20 \mu \mathrm{L}$ of completed Precipitation/ Inactivation buffer (Ambion) added. After recovery from precipitation, all samples were run on a $10 \%$ sequencing polyacrylamide gel in $0.5 \times$ TBE. Results were analyzed by phosphorimaging.

\section{Acknowledgments}

We thank Nicolas Villagra and Francesca Fiorini for participating in the experiments in Figures 2B and 4, respectively. Furthermore, we thank Francesca Fiorini for purifying Hfq and for technical assistance with the in vitro RNA analyses. We are very grateful to Jackie Plumbridge and Pepe Casadesús for critical reading of the manuscript and for insightful feedback. This work was made possible by a grant from the French National Research Agency (ANR; BLAN07-1_187785). 


\section{References}

Aiba H. 2007. Mechanism of RNA silencing by Hfq-binding small RNAs. Curr Opin Microbiol 10: 134-139.

Aiba H, Hanamura A, Yamano H. 1991. Transcriptional terminator is a positive regulatory element in the expression of the Escherichia coli crp gene. I Biol Chem 266: 17211727.

Babitzke P, Romeo T. 2007. CsrB sRNA family: Sequestration of RNA-binding regulatory proteins. Curr Opin Microbiol 10: 156-163.

Bertani G. 2004. Lysogeny at mid-twentieth century: P1, P2, and other experimental systems. J Bacteriol 186: 595-600.

Bossi L, Figueroa-Bossi N. 2007. A small RNA downregulates LamB maltoporin in Salmonella. Mol Microbiol 65: 799810.

Bouvier M, Sharma CM, Mika F, Nierhaus KH, Vogel J. 2008. Small RNA binding to $5^{\prime}$ mRNA coding region inhibits translational initiation. Mol Cell 32: 827-837.

Brennan RG, Link TM. 2007. Hfq structure, function and ligand binding. Curr Opin Microbiol 10: 125-133.

Brown L, Elliott T. 1996. Efficient translation of the RpoS $\sigma$ factor in Salmonella typhimurium requires host factor I, an RNA-binding protein encoded by the hfq gene. I Bacteriol 178: 3763-3770.

Chen S, Zhang A, Blyn LB, Storz G. 2004. MicC, a second smallRNA regulator of Omp protein expression in Escherichia coli. J Bacteriol 186: 6689-6697.

Cisneros B, Court D, Sanchez A, Montanez C. 1996. Point mutations in a transcription terminator, $\lambda \mathrm{tI}$, that affect both transcription termination and RNA stability. Gene 181: 127133.

Datsenko KA, Wanner BL. 2000. One-step inactivation of chromosomal genes in Escherichia coli K-12 using PCR products. Proc Natl Acad Sci 97: 6640-6645.

Ding Y, Davis BM, Waldor MK. 2004. Hfq is essential for Vibrio cholerae virulence and downregulates $\sigma$ expression. Mol Microbiol 53: 345-354.

Douchin V, Bohn C, Bouloc P. 2006. Down-regulation of porins by a small RNA bypasses the essentiality of the regulated intramembrane proteolysis protease RseP in Escherichia coli. J Biol Chem 281: 12253-12259.

Ellermeier CD, Janakiraman A, Slauch JM. 2002. Construction of targeted single copy lac fusions using $\lambda$ Red and FLPmediated site-specific recombination in bacteria. Gene 290: 153-161.

Escott GM, Adams DJ. 1995. Chitinase activity in human serum and leukocytes. Infect Immun 63: 4770-4773.

Figueroa-Bossi N, Coissac E, Netter P, Bossi L. 1997. Unsuspected prophage-like elements in Salmonella typhimurium. Mol Microbiol 25: 161-173.

Figueroa-Bossi N, Lemire S, Maloriol D, Balbontín R, Casadesús J, Bossi L. 2006. Loss of Hfq activates the $\sigma^{\mathrm{E}}$-dependent envelope stress response in Salmonella enterica. Mol Microbiol 62: 838-852.

Francetic O, Belin D, Badaut C, Pugsley AP. 2000. Expression of the endogenous type II secretion pathway in Escherichia coli leads to chitinase secretion. EMBO J 19: 6697-6703.

Geissmann TA, Touati D. 2004. Hfq, a new chaperoning role: Binding to messenger RNA determines access for small RNA regulator. EMBO J 23: 396-405.

Gottesman S, McCullen CA, Guillier M, Vanderpool CK, Majdalani N, Benhammou J, Thompson KM, FitzGerald PC, Sowa NA, FitzGerald DJ. 2006. Small RNA regulators and the bacterial response to stress. Cold Spring Harb Symp Quant Biol 71: 1-11.
Guarneros G, Portier C. 1991. Different specificities of ribonuclease II and polynucleotide phosphorylase in 3'mRNA decay. Biochimie 73: 543-549.

Guillier M, Gottesman S, Storz G. 2006. Modulating the outer membrane with small RNAs. Genes \& Dev 20: 2338-2348.

Guisbert E, Rhodius VA, Ahuja N, Witkin E, Gross CA. 2007. Hfq modulates the $\sigma^{\mathrm{E}}$-mediated envelope stress response and the $\sigma^{32}$-mediated cytoplasmic stress response in Escherichia coli. J Bacteriol 189: 1963-1973.

Kachroo AH, Kancherla AK, Singh NS, Varshney U, Mahadevan S. 2007. Mutations that alter the regulation of the chb operon of Escherichia coli allow utilization of cellobiose. Mol Microbiol 66: 1382-1395.

Kaspar S, Bott M. 2002. The sensor kinase CitA (DpiB) of Escherichia coli functions as a high-affinity citrate receptor. Arch Microbiol 177: 313-321.

Keyhani NO, Roseman S. 1997. Wild-type Escherichia coli grows on the chitin disaccharide, $\mathrm{N}, \mathrm{N}^{\prime}$-diacetylchitobiose, by expressing the cel operon. Proc Natl Acad Sci 94: 1436714371.

Keyhani NO, Li XB, Roseman S. 2000. Chitin catabolism in the marine bacterium Vibrio furnissii. Identification and molecular cloning of a chitoporin. J Biol Chem 275: 33068-33076.

Lapouge K, Schubert M, Allain FH, Haas D. 2008. Gac/Rsm signal transduction pathway of $\gamma$-proteobacteria: From RNA recognition to regulation of social behaviour. Mol Microbiol 67: 241-253.

Lemire S, Figueroa-Bossi N, Bossi L. 2008. A singular case of prophage complementation in mutational activation of recET orthologs in Salmonella enterica serovar Typhimurium. J Bacteriol 190: 6857-6866.

Lutgens M, Gottschalk G. 1980. Why a co-substrate is required for anaerobic growth of Escherichia coli on citrate. I Gen Microbiol 119: 63-70.

Majdalani N, Cunning C, Sledjeski D, Elliott T, Gottesman S. 1998. DsrA RNA regulates translation of RpoS message by an anti-antisense mechanism, independent of its action as an antisilencer of transcription. Proc Natl Acad Sci 95: 1246212467.

Majdalani N, Vanderpool CK, Gottesman S. 2005. Bacterial small RNA regulators. Crit Rev Biochem Mol Biol 40: 93113.

Maloy SR, Roth JR. 1983. Regulation of proline utilization in Salmonella typhimurium: Characterization of put:Mu d(Ap, lac) operon fusions. J Bacteriol 154: 561-568.

Mandin P, Gottesman S. 2009. A genetic approach for finding small RNAs regulators of genes of interest identifies RybC as regulating the DpiA/DpiB two-component system. Mol Microbiol 72: 551-565.

Massé E, Gottesman S. 2002. A small RNA regulates the expression of genes involved in iron metabolism in Escherichia coli. Proc Natl Acad Sci 99: 4620-4625.

Massé E, Escorcia FE, Gottesman S. 2003. Coupled degradation of a small regulatory RNA and its mRNA targets in Escherichia coli. Genes \& Dev 17: 2374-2383.

McClelland M, Sanderson KE, Spieth J, Clifton SW, Latreille P, Courtney L, Porwollik S, Ali J, Dante M, Du F, et al. 2001. Complete genome sequence of Salmonella enterica serovar Typhimurium LT2. Nature 413: 852-856.

Miller JH. 1992. A short course in bacterial genetics. A laboratory manual and handbook for Escherichia coli and related bacteria. Cold Spring Harbor Laboratory Press, Cold Spring Harbor, NY.

Mizuno T, Chou MY, Inouye M. 1984. A unique mechanism regulating gene expression: Translational inhibition by 
a complementary RNA transcript (micRNA). Proc Natl Acad Sci 81: 1966-1970.

Moll I, Afonyushkin T, Vytvytska O, Kaberdin VR, Bläsi U. 2003. Coincident Hfq binding and RNase E cleavage sites on mRNA and small regulatory RNAs. RNA 9: 1308-1314.

Morita T, Maki K, Aiba H. 2005. RNase E-based ribonucleoprotein complexes: Mechanical basis of mRNA destabilization mediated by bacterial noncoding RNAs. Genes \& Dev 19: 2176-2186.

Morita T, Mochizuki Y, Aiba H. 2006. Translational repression is sufficient for gene silencing by bacterial small noncoding RNAs in the absence of mRNA destruction. Proc Natl Acad Sci 103: 4858-4863.

Mott JE, Galloway JL, Platt T. 1985. Maturation of Escherichia coli tryptophan operon mRNA: Evidence for $3^{\prime}$ exonucleolytic processing after rho-dependent termination. EMBO J 4: 1887-1891.

Padalon-Brauch G, Hershberg R, Elgrably-Weiss M, Baruch K, Rosenshine I, Margalit H, Altuvia S. 2008. Small RNAs encoded within genetic islands of Salmonella typhimurium show host-induced expression and role in virulence. Nucleic Acids Res 36: 1913-1927.

Papenfort K, Pfeiffer V, Mika F, Lucchini S, Hinton JC, Vogel J. 2006. $\sigma^{\mathrm{E}}$-Dependent small RNAs of Salmonella respond to membrane stress by accelerating global omp mRNA decay. Mol Microbiol 62: 1674-1688.

Plumbridge J, Pellegrini O. 2004. Expression of the chitobiose operon of Escherichia coli is regulated by three transcription factors: NagC, ChbR and CAP. Mol Microbiol 52: 437-449.

Rasmussen AA, Eriksen M, Gilany K, Udesen C, Franch T, Petersen C, Valentin-Hansen P. 2005. Regulation of ompA mRNA stability: The role of a small regulatory RNA in growth phase-dependent control. Mol Microbiol 58: 14211429.

Rasmussen AA, Johansen J, Nielsen JS, Overgaard M, Kallipolitis B, Valentin-Hansen P. 2009. A conserved small RNA promotes silencing of the outer membrane protein YbfM. Mol Microbiol 72: 566-577.

Santiviago CA, Toro CS, Hidalgo AA, Youderian P, Mora GC. 2003. Global regulation of the Salmonella enterica serovar typhimurium major porin, OmpD. I Bacteriol 185: 59015905.

Schmieger H. 1972. Phage P22-mutants with increased or decreased transduction abilities. Mol Gen Genet 119: 75-88.

Seitz H. 2009. Redefining MicroRNA targets. Curr Biol 19: 1-4.

Sittka A, Pfeiffer V, Tedin K, Vogel J. 2007. The RNA chaperone $\mathrm{Hfq}$ is essential for the virulence of Salmonella typhimurium. Mol Microbiol 63: 193-217.

Soper TJ, Woodson SA. 2008. The rpoS mRNA leader recruits Hfq to facilitate annealing with DsrA sRNA. RNA 14: 19071917.

Storz G, Opdyke JA, Zhang A. 2004. Controlling mRNA stability and translation with small, noncoding RNAs. Curr Opin Microbiol 7: 140-144.

Thompson KM, Rhodius VA, Gottesman S. 2007. $\sigma^{\mathrm{E}}$ Regulates and is regulated by a small RNA in Escherichia coli. I Bacteriol 189: 4243-4256.

Udekwu KI, Wagner EG. 2007. $\sigma^{\mathrm{E}}$ Controls biogenesis of the antisense RNA MicA. Nucleic Acids Res 35: 1279-1288.

Udekwu KI, Darfeuille F, Vogel J, Reimegard J, Holmqvist E, Wagner EG. 2005. Hfq-dependent regulation of OmpA synthesis is mediated by an antisense RNA. Genes \& Dev 19: $2355-2366$.

Uzzau S, Figueroa-Bossi N, Rubino S, Bossi L. 2001. Epitope tagging of chromosomal genes in Salmonella. Proc Natl Acad Sci 98: 15264-15269.
Valentin-Hansen P, Johansen J, Rasmussen AA. 2007. Small RNAs controlling outer membrane porins. Curr Opin Microbiol 10: 152-155.

Vogel J. 2009. A rough guide to the non-coding RNA world of Salmonella. Mol Microbiol 71: 1-11.

Vogel J, Papenfort K. 2006. Small non-coding RNAs and the bacterial outer membrane. Curr Opin Microbiol 9: 605-611.

Vogel J, Bartels V, Tang TH, Churakov G, Slagter-Jager JG, Huttenhofer A, Wagner EG. 2003. RNomics in Escherichia coli detects new sRNA species and indicates parallel transcriptional output in bacteria. Nucleic Acids Res 31: 64356443.

Wassarman KM, Repoila F, Rosenow C, Storz G, Gottesman S. 2001. Identification of novel small RNAs using comparative genomics and microarrays. Genes \& Dev 15: 1637-1651.

Waters LS, Storz G. 2009. Regulatory RNAs in bacteria. Cell 136: 615-628.

Yamamoto K, Matsumoto F, Minagawa S, Oshima T, Fujita N, Ogasawara N, Ishihama A. 2009. Characterization of CitACitB signal transduction activating genes involved in anaerobic citrate catabolism in Escherichia coli. Biosci Biotechnol Biochem 73: 346-350.

Yu D, Ellis HM, Lee EC, Jenkins NA, Copeland NG, Court DL. 2000. An efficient recombination system for chromosome engineering in Escherichia coli. Proc Natl Acad Sci 97: 59785983.

Zhang A, Wassarman KM, Ortega J, Steven AC, Storz G. 2002. The Sm-like Hfq protein increases OxyS RNA interaction with target mRNAs. Mol Cell 9: 11-22.

Zhang A, Wassarman KM, Rosenow C, Tjaden BC, Storz G, Gottesman S. 2003. Global analysis of small RNA and mRNA targets of Hfq. Mol Microbiol 50: 1111-1124.

Zhu Z, Zheng T, Homer RJ, Kim YK, Chen NY, Cohn L, Hamid Q, Elias JA. 2004. Acidic mammalian chitinase in asthmatic Th2 inflammation and IL-13 pathway activation. Science 304: 1678-1682. 


\section{Erratum}

Genes \& Development 23: 2004-2015 (2009)

\section{Caught at its own game: regulatory small RNA inactivated by an inducible transcript mimicking its target}

Nara Figueroa-Bossi, Martina Valentini, Laurette Malleret, and Lionello Bossi

For the above paper, the French Centre National de la Recherche Scientifique (CNRS) has requested that the name of Francesca Fiorini, whose involvement in this study is described in the acknowledgment section of the above article, be included in the author list.

The new author list is as follows:

Nara Figueroa-Bossi, Martina Valentini, Laurette Malleret, Francesca Fiorini and Lionello Bossi

Francesca Fiorini's present address is Department of Biology, ENS, 46 rue d'ULM, 75005 Paris, France. 


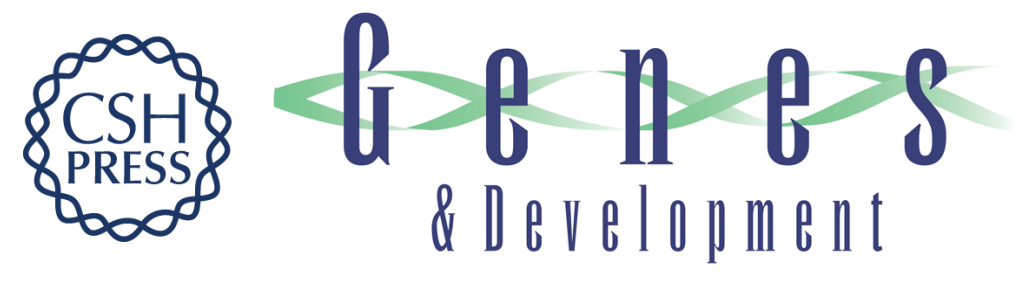

\section{Caught at its own game: regulatory small RNA inactivated by an inducible transcript mimicking its target}

Nara Figueroa-Bossi, Martina Valentini, Laurette Malleret, et al.

Genes Dev. 2009, 23: originally published online July 28, 2009

Access the most recent version at doi:10.1101/gad.541609

\section{Supplemental http://genesdev.cshlp.org/content/suppl/2009/08/14/23.17.2004.DC1 \\ Material}

Related Content Regulating the regulator: an RNA decoy acts as an OFF switch for the regulation of an SRNA

Pierre Mandin and Susan Gottesman

Genes Dev. September , 2009 23: 1981-1985 Erratum: Caught at its own game:

regulatory small RNA inactivated by an inducible transcript mimicking its target

Nara Figueroa-Bossi, Martina Valentini, Laurette Malleret, et al.

Genes Dev. April , 2010 24: 734

References This article cites 68 articles, 31 of which can be accessed free at:

http://genesdev.cshlp.org/content/23/17/2004.full.html\#ref-list-1

Articles cited in:

http://genesdev.cshlp.org/content/23/17/2004.full.html\#related-urls

\section{License}

Email Alerting

Receive free email alerts when new articles cite this article - sign up in the box at the top Service

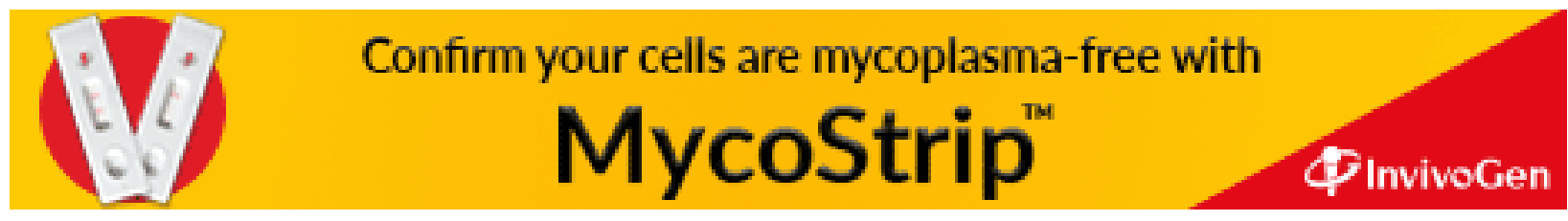

